\title{
Problemática de la educación ambiental en las instituciones educativas
}

\section{Problematic of the Environmental Education in Educational Institutions}

\section{Problemática da Educação Ambiental em Instituições de Ensino}

\author{
Liliana Hayde Gutiérrez Sabogal ${ }^{1}$
}

Fecha de recepción: abril 2015

Fecha de aceptación: diciembre 2015

Para citar este artículo: Gutiérrez, L. (2015). Problemática de la educación ambiental en las instituciones educativas. Revista Científica, 23, 57-76. Doi: $\underline{10.14483 / \text { distrital.jour.RC.2015.23.a5 }}$

\section{Resumen}

El presente artículo bosqueja la comprensión de la actual situación de la educación ambiental en las Instituciones Educativas de Colombia, teniendo en cuenta los aspectos que a nuestro parecer parecen incidir en esta problemática y las posibles interrelaciones que entre ellas se pueden evidenciar, como la primera etapa de la investigación doctoral dirigida por el doctor Francisco González.

Palabras Clave: Educación Ambiental, Legislación Ambiental, Problemática Educativa.

\section{Abstract}

The following article sketches the understanding of the actual situation of environmental education in Colombian educational institutions, taking in to account the aspects that seem to have an impact on this problematic and the possible interrelationships between them like the first stage of the doctoral research lead by Doctor Francisco Gonzalez.

Keywords: environmental education, teaching and learning, educational problematic.

\section{Resumo}

O artigo que se segue esboça a compreensão da situação atual da educação ambiental nas instituições de ensino da Colômbia, levando em conta os aspectos que parecem ter um impacto sobre esta problemática e as possíveis inter-relações entre eles como o primeiro estágio da liderança pesquisa de doutorado pelo doutor Francisco González.

Palavras-chave: educação ambiental, ensino e aprendizagem, problemática educacional. 


\section{Introducción}

Durante los años, y a través de la experiencia docente, se ha percibido la falta de relación que existe entre los contenidos escolares de educación ambiental (EA) manejados en el área de ciencias naturales y las actitudes, aptitudes, valores y comportamientos de los alumnos. El trabajo en el aula y las actividades pedagógicas y lúdicas relacionadas con la EA, tales como proyectos del medio ambiente institucional desarrolladas durante varios años lectivos, no evidencian la apropiación de los alumnos a través de sus acciones y, por el contrario, se siguen requiriendo actividades como recolección de papeles y basura después del descanso, campañas de aseo rutinarias, cuidado de jardines, campañas anti ruido, recordación constante de recolección y reciclaje, reducción de desperdicio, uso adecuado del agua, entre otras. Lo anterior, proporciona la certeza de que la EA en las instituciones educativas no ha logrado sus objetivos en concordancia con lo planeado, situación que también ha sido explicada por algunos investigadores quienes concluyen que:

las intervenciones educativas son procesos lentos y progresivos que no producen cambios inmediatos en los sujetos, no resulta fácil poner de manifiesto la existencia de una cierta relación directa entre la mejora de ciertas condiciones ambientales o la disminución de determinados problemas ecológicos y la realización de una determinada intervención educativa. (Navarro, 2007, p. 36)

Este escenario local influye en problemáticas y soluciones globales de la sociedad y la humanidad. Actualmente, se habla mucho del calentamiento global, la ruptura de la capa de ozono; la extinción de especies; la erosión, la degradación y el uso indebido del suelo; la contaminación de la biosfera y el agua; la destrucción de páramos y áreas de bosque natural; la extracción de minerales, etc. Una multitud de actividades e intervenciones del hombre en la naturaleza sin reflexión ni acción alguna que proyecte la disminución de las consecuencias de su fisgoneo, ni el desarrollo de actitudes y valores ecológicos que pretendan mejorar la situación. Lo anterior se traduce en la frase "pensar globalmente, actuar localmente" y el comentario de Ibrahim (2007): "la solución a los problemas medioambientales globales solo se traducirá en acciones concretas cuando se tomen en consideración las diferencias culturales, ecológicas y económicas del medio ambiente local".

En definitiva, si este escenario continúa en forma progresiva la calidad de vida de todos los seres que compartimos el planeta seguirá disminuyendo, haciendo que muchas acciones sociales se perfilen en atender realidades que pueden evitarse si se actúa de forma coherente y amigable con el entorno desde ahora. Entonces, la promoción y la prevención pueden ser la forma más barata y evidente de atacar problemas relacionados con el medio ambiente.

\section{Factores que inciden en la problemática}

Desde la experiencia docente, los factores que podrían estar incidiendo en la brecha que existe entre los contenidos escolares de EA y las acciones por parte de los alumnos se relacionan con aspectos del contexto escolar, la institución educativa, profesores, padres de familia y los mismos alumnos, sin olvidar la normatividad al respecto; aspectos que requieren ser objeto de reflexión e investigación pedagógica con el propósito de conocer la complejidad de la realidad y formar ciudadanos comprometidos con la preservación del ambiente, conscientes de su acción y la transformación de la sociedad hacia un modelo que garantice mejores condiciones de vida para todas las formas vivientes del planeta.

\section{Contexto escolar}

El entorno en el cual los niños, niñas y jóvenes están inmersos recrea su formación y permite establecer algunas características de su idiosincrasia que 
inciden en la problemática analizada. El contexto escolar se refiere no solamente al espacio escolar sino a los padres de familia, el sector productivo, la administración municipal y la comunidad en la cual viven los alumnos; actores y entidades en donde los comportamientos amigables con el ambiente tienen poca importancia.

Diversos autores coinciden en la importancia del contexto en la educación: "la vida humana es siempre vida en contexto" y es evidente que se "necesita una profunda reflexión sobre nuestros condicionamientos, de nuestra visión temporal, espacial, y de relaciones sociales y temáticas [para lograr relacionar] territorio, espacio y trama social" (Martínez, 2007).

Parece ser que el entorno familiar, es decir, los padres y familiares de los alumnos no se preocupan por generar cultura ecológica en sus casas y no se empoderan de la obligación que tienen en la formación de sus hijos de acuerdo a la Constitución Política de Colombia y a su inherente función en la sociedad. Para ellos no es importante la EA como formación básica, ni la transformación y el cambio que se puede evidenciar en el hogar y en las actividades productivas y cotidianas en pro de la conservación y preservación del medio ambiente. Es así como muchas actividades se ejecutan sin pensar en el daño que se le está haciendo al entorno: permanentemente se queman las basuras, se botan desperdicios en las quebradas, no se separan basuras en las casas, el uso del agua es inadecuado y existe muy poca reflexión acerca de los daños individuales que como seres humanos realizamos diariamente.

El sector productivo de la sociedad actual está impregnado de características propias de la modernidad y del capitalismo que hace que por encima de la conservación de los recursos naturales y la relación amistosa entre el hombre y las demás formas de vida se establezcan patrones propios del mercado en donde la productividad y el rendimiento de las industrias no consideran el desarrollo sin poner en riesgo la capacidad del planeta para sostener a las generaciones futuras.
Esto acontece con el entorno familiar y el sector productivo, pero parece que dentro de las instituciones educativas la cuestión no es diferente: se llevan a cabo actividades que no tienen implicaciones más allá del aula de clase, "la evolución realizada por el alumno en un contexto de aprendizaje concreto en muchos casos no se mantiene al cambiar de contexto", posible explicación a la falta de apropiación de los conocimientos ambientales de los estudiantes que evidencien cambios dentro del hogar o las comunidades (Cano, 2007), situación que se debe investigar para poder dar alternativas de solución que se ampliarán más adelante.

Si se analiza la situación desde las administraciones públicas locales y regionales, parece no haber una directriz institucional, municipal o regional a partir de la cual se desarrollen estrategias de EA consolidadas, lo cual es consistente con la apreciación de Solís (2007), quien afirma que existe una "educación ambiental muy sectorizada, donde las entidades y organismos trabajan de forma independiente sin un hilo conductor que permita desarrollar una acción educativa en una comarca determinada" teniendo en cuenta las características y necesidades del contexto.

Desde esta realidad es necesario desarrollar conciencia ciudadana con el propósito de aportar a la situación regional y mundial a través de la escuela, pues es la institución educativa por excelencia que "hace parte del problema, pero a la vez la alternativa desde donde se recupera y crearan valores que posibiliten el desarrollo de las comunidades" (López, 1994).

Se han publicado experiencias exitosas que logran enmarcar interrelaciones e hilos conductores entre diversas instituciones como universidades, administraciones municipales e instituciones educativas públicas. Por ejemplo:

en el municipio de Planeta Rica en Córdoba se construyeron alternativas que permiten asumir el diálogo de saberes como enfoque pedagógico válido, que permitan desarrollar procesos pedagógicos por proyectos para articular la práctica educativa 
cotidiana a los procesos de planeamiento y desarrollo local, que permitan elaborar en forma participativa entre estudiantes y docentes textos escolares, contextualizados en la cultura regional y que permitan a estudiantes construir soluciones planeadas a los problemas teóricos y teórico-prácticos que surjan de los contenidos que se estudian en la escuela o colegio. (Equipo de trabajo, 1994 p. 208)

\section{El anterior ejemplo muestra cómo}

Las innovaciones que antes eran esfuerzos aislados y frustrados, han ido cobrando vida como aportes valioso en el nuevo proceso de reestructuración, que ahora se hace dentro del Proyecto Educativo Institucional, PEl. Esto posibilita que las innovaciones educativas en general y pedagógicas en particular, cobren vida en el proceso de desarrollo del establecimiento educativo. Quienes antes se mostraban reacios al cambio están participando ahora acuciosamente en la recuperación de las experiencias de cada quien y en la búsqueda de alternativas que aireen la práctica educativa y pedagógica rutinizada con currículos elaborados tradicionalmente. (Equipo de trabajo, 1994, pp. 209-210)

Es pues un ejemplo claro de articulación del PEI de una institución educativa pública con el Plan de Desarrollo Educativo Municipal, que a su vez es tenido en cuenta por la Administración en el Plan de Inversión.

En todo caso, el contexto debe ser el punto de partida de la EA:

los proyectos son factibles de plantear desde una unidad programática, desde un tema, desde una situación problemática. Lo fundamental es que correspondan al contexto cultural, social y natural en el que se desarrollan y busquen la integración con el ánimo de que su proyección tenga incidencia directa en la formación integral del niño y lo preparen para actuar, consiente y responsablemente, en el manejo de su entorno. (Carrasco, 1994, p.110)
En otras palabras, "Ios proyectos escolares en educación ambiental deben estar inscritos en la problemática ambiental local, regional y/o nacional, a través de la concertación con las entidades que de una u otra manera, están comprometidas en la búsqueda de soluciones" (Giordan y Souchon, 1997), enfocados a la acción escolar educativa, la formación de ciudadanos con actitudes, aptitudes y valores, que al salir del contexto escolar logren interesarse e interrelacionarse con la comunidad y así convertirse en participes de las actividades de la comunidad.

En conclusión, el contexto escolar es un espacio que requiere ser aprovechado para activar mecanismos de participación ciudadana que desde los hogares, instituciones educativas, administración pública y sector productivo pueden interrelacionarse para hacer que la acción educativa ambiental escolar salga de las aulas y transforme entornos.

\section{La institución educativa}

Como se dijo anteriormente, la institución educativa es parte del contexto escolar y como un espacio de formación importante en la apropiación del conocimiento escolar en EA es el lugar por excelencia para la integración de una planeación transversal que involucre la administración municipal, el PEI, los estamentos de la institución educativa y las áreas del conocimiento dentro de una malla curricular coherente que interrelacione el contexto particular de cada comunidad, determine estrategias didácticas y eduque a los alumnos en comportamientos amigables con el medio ambiente.

Pero la percepción de lo que está ocurriendo en el proceso de EA dentro de la escuela se relaciona con un activismo y reduccionismo en cuanto se da cumplimientos a los lineamientos estatales como el PRAE (Proyectos Ambiental Escolar), proyecto del medio ambiente y los lineamientos del Ministerio de Educación Nacional (MEN) pero de una manera no interdisciplinar ni acordes con las necesidades regionales y se ha convertido en una reunión de actividades relacionadas con el medio ambiente 
que no empoderan a los alumnos para transformar su entorno, entre otras razones porque no se relacionan directamente con las problemáticas locales.

La EA en las instituciones educativas se ha convertido en una tarea específica del área de ciencias naturales con una programación que no delimita ni enfatiza en contenidos escolares que logren desarrollar en los alumnos cambios en pro del ambiente. No se trabaja con la posibilidad de proyección hacia la comunidad con objetivos claros acerca de la formación en actitudes, aptitudes, hábitos y comportamientos ambientales y maneja proyectos ambientales dentro de cada escuela a escala micro, dirigidos o mandados desde el área de ciencias naturales sin buscar una relación con las posibilidades externas más cercanas, es decir, no parten del conocimiento cotidiano de la comunidad. Generalmente se incluyen temáticas en las asignaturas de biología, física y/o química como actividades adicionales o complementarias, pero descontextualizadas, por ejemplo: hablar de la contaminación de la biosfera en química como una exposición con carácter evaluativo, de las implicaciones del uso del suelo en física cuando se trate el tema de propiedades físicas de la materia o de la contaminación de los ríos y el uso inadecuado del agua cuando se abre la discusión de los ecosistemas acuáticos en biología. Esto refleja el reduccionismo y la poca importancia que dentro del plan de estudios se le confiere a la EA, así como la falta de un hilo conductor y una transversalidad tan necesaria para lograr la apropiación de los contenidos escolares en los alumnos.

A pesar de que teóricamente el PEI es un puente entre la escuela y el contexto, en la realidad no se evidencia claramente: la formación de un perfil específico emanado desde la institución educativa y los requerimientos de la sociedad específica no son convergentes.

la escuela se pone de espaldas a la realidad, por ejemplo, cuando a un niño que vive en una zona de alta contaminación (ladrillera en Bogotá), se le enseñan, en el aula de clase, las definiciones de la misma, obligándolo a repetirlas y a memorizarlas, sin ninguna contextualización, solamente para resolver un examen, olvidándose que él hace parte de una comunidad que sufre el problema y negándole la posibilidad de influir sobre él y transformándolo." (Carrasco, 1994, p. 109).

Entonces, se requiere de un trabajo interinstitucional, interdisciplinario y de un dialogo de saberes. Las actividades escolares como el PEI y los proyectos de medio ambiente de las instituciones educativas no están pensados dentro de un marco de EA, ni atendiendo problemáticas especificas con alternativas didácticas innovadoras dejando de percibir la importancia de los saberes de los estudiantes y la comunidad educativa así como sus percepciones, necesidades y problemáticas ambientales que deberían ser los pilares de la programación de contenidos en la formación ambiental.

En la misma línea, no existe una manera de relacionar las actividades que desde las distintas áreas se realizan o desde los proyectos que la ley exige se desarrollan, para lograr la interdisciplinariedad y transversalidad de las áreas del conocimiento que fluyan hacia el perfil del estudiante que se quiere formar. Por ello, se peca en exceso de actividades y poca reflexión pedagógica quedando la EA como una serie de intentos de interrelacionar los conocimientos escolares de tal forma que sean útiles en el momento en que se quiera solucionar problemas cotidianos. Lo anterior conIleva a la situación muy bien descrita:

El activismo supone un simulacro de aprendizaje significativo: se responden las preguntas sin apenas elaboración de las respuestas, se pasa en todo momento de una tarea a otra sin un instante de reflexión, se manifiestan hipótesis sin que esté muy claro el por qué se hace o se hace todo de prisa, como si lo importante fuera «cubrir el expediente», es decir, que se ha realizado la tarea propuesta. Como no hay un protagonismo real de los alumnos, estos "pueden responder a las preguntas como si de adivinar un juego se tratara». (García \& Cano, 2006, p.128) 
La anterior preocupación permite reflexionar acerca de la EA, no como parte del área de ciencias naturales nada más, sino que puede ser pensada desde la transversalidad con otras áreas y tipos de conocimiento y proyectos diferentes. Es un hecho que el área de ciencias sociales alcanza a tocar estos temas pero de manera aislada y sin pretensiones ambiciosas ni interrelacionando los conocimientos teóricos con la praxis. En este sentido, es necesario concebir el papel de la EA como "el desarrollo de un proceso que formule nuevas lecturas de mundo, mediante la comprensión y vivencia planetaria, con el objetivo de la diseminación de una conciencia en pro del desarrollo integral que implique todas las esferas de la vida.", lo cual implica el abordaje de la EA desde un dialogo de saberes entre las disciplinas bajo parámetros de contextualización y reconceptualización con enfoque científico y por las diversas áreas del conocimiento (Fonseca, 2007).

\section{En todo caso, los proyectos se consideran}

Una posibilidad [...] que desde el aula de clases y desde la institución educativa se vinculen a la solución de la problemática particular de una localidad o región, dado que ello posibilita la generación de espacios comunes de reflexión en los que desarrollan criterios de solidaridad, tolerancia, búsqueda del consenso, autonomía y creatividad y que, en ultimas e igualmente, preparan para la autogestión en la búsqueda de un mejoramiento de la calidad de vida, propósito último de la educación ambiental. (Carrasco, 1994, p. 109)

Es importante, entonces, tener en cuenta estos factores cuando se trata de planear, ejecutar y evaluar los proyectos que se llevan a cabo dentro de las instituciones educativas.

De hecho, se ha demostrado mediante investigaciones que proyectos de medio ambiente institucionales pueden aumentar el compromiso ambiental de los sujetos, por ejemplo, Chamorro (2007) reporta un estudio de caso en Argentina donde "los datos analizados muestran un importante aumento del compromiso ambiental de los egresados en función de la maduración del proyecto educativo de la EAN (Escuela Argentina de Naturalistas), aunque esta diferencia entre las medias no lleguen a ser significativas." (Chamorro, 2007, p. 145).

Si se echa un vistazo al currículo, Sacristán (2010) lo define como:

Una pasarela entre la cultura y la sociedad exteriores a las instituciones educativas, por un lado, y la cultura de los sujetos, por el otro; entre la sociedad que hoy es y la que habrá mañana, entre las posibilidades de conocer, de saber comunicar y expresarse en contraposición a la cerrazón y a la ignorancia. (Sacristán, 2010, p. 12)

Por lo anterior y porque:

La inclusión de la dimensión ambiental en el currículo a partir de proyectos permite integrar las diversas áreas del conocimiento para el manejo de un universo conceptual aplicado a la solución de problemas ... explorar cual es la participación de cada una de las disciplinas, en un trabajo interdisciplinario y/o transdisciplinario que posibilita la formación en la ciencia, la técnica y la tecnología desde un marco social que sea el referente de identidad del individuo y que genere un compromiso con el mismo y con su comunidad ... permite la exploración de las posibilidades de un trabajo interinstitucional, atravesado por el concepto de gestión y la claridad de competencias y responsabilidades. (Carrasco, 1994, p. 109)

Esto supone la tarea de tejer una red interdisciplinaria curricular en la EA desde la reflexión y la interdisciplinariedad entendida como el trabajo desde cada una de las disciplinas en torno a una actividad concreta, el razonar desde cada una de las disciplinas para la comprensión de una problemática o situación particular, la puesta en común de los diversos puntos de vista, las diversas 
perspectivas, los diversos saberes, en torno al fenómeno, y la búsqueda de consenso que permita el avance en la explicación estos y no como la sumatoria de actividades. Desde esta perspectiva cada una de las disciplinas mantendrá sus dinámicas y hará sus propias reflexiones contribuyendo a la solución de problemas, evolucionando y haciendo evolucionar a las otras disciplinas, en un terreno interdisciplinario, generado por el problema o situación que se trabaja (Carrasco, 1994, p.112).

En este sentido, es importante recapacitar acerca de las diversas propuestas pedagógicas que se han investigado y depurado y que pueden aportar al desarrollo de un currículo pertinente con la situación del entorno que mejoren la formación ambiental de los alumnos con eficacia y eficiencia en las instituciones educativas en cuanto:

- Las intenciones educativas (objetivos) deben darse en los campos abarcados por contenidos conceptuales, procedimentales y actitudinales. Los contenidos deben ser significativos en la perspectiva local y global, la construcción del conocimiento debe darse a partir de las concepciones previas de los sujetos, como punto de partida y, el conocimiento elaborado, como punto de Ilegada, en la dinámica constante con que el conocimiento históricamente se produce. Ellas orientan una metodología que esté presente en la transversalidad de los contenidos científicos, socioculturales, éticos y políticos. (Fonseca, 2007, p. 87).

- El debate sobre las cuestiones ambientales debe superar la retórica del discurso y pasar para la acción, poniendo los actores sociales en la condición de corresponsables por la mejoría de la calidad de vida en el planeta, considerando la dimensión política de la educación ambiental en la construcción de la ciudadanía. De ahí que haya la necesidad de la implementación de esa temática, como fue propuesta al alcance. (Fonseca, 2007, p. 87)

- Transversalidad entre las disciplinas en una malla curricular en donde se admiten dos modalidades básicas de transversalidad: una de carácter interdisciplinar (en donde se considera a la EA como un área del conocimiento con entidad propia donde precipitan aportaciones del resto de disciplinas) y otra de carácter multidisciplinar (la EA pierde su carácter de materia específica para convertirse en materia integrada que invade todas las esferas del conocimiento). (Martínez, 2007, p. 121)

- Las estrategias de corte constructivistas pueden promover una interacción del alumno con los contenidos culturales y escolares en busca de un aprendizaje significativo, si se quieren alcanzar logros más profundos, basado en un contexto real y con sentido para quien aprende. (Escalante, 2007, p. 104)

\section{Profesores:}

Uno de los actores que inciden en cualquier proceso de formación es el profesor y por eso requiere un análisis amplio. Son diversos los aspectos que están relacionados con su influencia en la EA que se evidencian claramente en el momento de desarrollar y evaluar proyectos y alternativas de solución en diversas dimensiones. A continuación, se describen algunos aspectos que podrían estar incidiendo en la problemática enunciada al hacer cierto análisis y contrastando con algunas investigaciones que se han realizado al respecto:

La formación profesional de los docentes es diversa y no están dirigida hacia la enseñanza de la EA; a pesar de ello, en su diario quehacer se evidencia y puede relacionarse en forma negativa en cuanto no permita delinear claramente lo que se quiere lograr en el aula o en la institución de acuerdo con el perfil del alumno deseado y los objetivos de la educación ambiental. En otras palabras, los docentes a cargo de la EA en las instituciones educativas tienen diversas formaciones profesionales, tal vez muy académicas, o con una especialidad específica (licenciados, microbiólogos, bacteriólogos, biólogos, ingenieros agropecuarios), que puede no contener la conceptualización, actitudes, 
aptitudes y comportamientos amigables con el ambiente y cuya consecuencia es una mirada más conceptual que practica de la naturaleza.

Lo que ocurre es que, por su procedencia académica, estos profesionales suelen conocer mejor los problemas ambientales o la realidad natural que los modelos y las teorías didácticas, carecen de un conocimiento teórico-práctico vinculado a la acción, y no tienen teorías mediadoras entre las declaraciones ideológicas y los grandes fines, ni entre los de la fundamentación teórica y la actuación educativa (García \& Cano, 2006).

Tal vez no existe un equilibrio entre la práctica y la teoría, entre la experiencia y el marco conceptual entonces; se puede caer en dos reduccionismos antagónicos: o bien sobrevaloramos la experiencia - por estar demasiado distantes las teorías - y educamos sin un marco teórico de referencia, o bien trasladamos de manera directa los planteamientos teóricos a la acción, menospreciando la práctica. (García \& Cano, 2006).

Investigaciones realizadas por Cano (2007) han mostrado que los docentes de aula "en su gran mayoría carecen de un marco conceptual y metodológico sobre la educación ambiental" relacionada con los ecosistemas propios del contexto en los cuales realizan su intervención y por ello no se desarrollan "actividades educativas que motiven en sus educandos los conocimientos, las actitudes y aptitudes para promover el manejo y gestión sostenible". Además, muestran carencia en su formación sobre la EA por lo que se les dificulta incorporar estos conceptos en su labor cotidiana" y carencia del "profesorado de conocimientos necesarios sobre los recursos pedagógicos que ofrece la educación ambiental y sobre el conocimiento de las potencialidades del desarrollo del ecosistema especifico".

Por otro lado, los supuestos epistemológicos propios de cada docente y su visión del mundo orientan su práctica profesional, develando una serie de pensamientos, actuaciones y discursos que son percibidos por los alumnos (currículo oculto) y crean en ellos ciertos imaginarios con los cuales desarrollan su formación haciendo que no se creen lazos entre el medio natural y los hábitos ecológicos porque tampoco existen estos en muchos docentes. A Algunos les parece que la evolución del universo hará que los daños que se están produciendo en el ambiente creen mecanismos de selección natural que desembocarán en la adaptación de las especies, de acuerdo a lo que ha venido ocurriendo en el planeta según la teoría de la evolución más aceptada. Otros creen que se deben realizar esfuerzos a nivel macro o Estatales para que las cosas cambien y que se debe esperar a que las instituciones gubernamentales o países desarrollados cambien de actitud y hagan algo porque de otra manera los intentos personales de nada han de servir al compararnos con lo poco que somos ante la inmensidad del universo.

Los anteriores factores hacen que los planes de estudio se delineen hacia ciertas preferencias, al igual que el desarrollo de las sesiones en el aula, originando en algunos casos incoherencia entre los proyectos de medio ambiente que se acompañan en cada curso relacionándolos más con las particularidades de cada profesor, que con las necesidades de los alumnos o la institución. De esta forma, cada docente acompaña una asignatura del plan de estudios y de una u otra manera inciden en la formación ambiental de los alumnos, pero no con los mismos objetivos de formación deseable o las características del contexto. La falta de congruencia con los objetivos de la EA, metodologías, planes, desarrollo de actividades, participación en proyectos y didácticas en el aula implican que se desaprovechen oportunidades para motivar y desarrollar las actitudes, aptitudes y valores en los alumnos a través de la interdisciplinariedad y transversalidad de dichos proyectos.

Adicionalmente, las concepciones del docente acerca de la construcción del conocimiento escolar en EA influyen en las didácticas y procedimientos pedagógicos que utilice durante las sesiones dedicadas a este proceso. 
Para muchos educadores ambientales la modificación de las ideas y de las conductas se consigue informando adecuadamente, transmitiendo sin más las «verdades» proambientales, como si en las personas que aprenden no hubiera concepciones resistentes al cambio que requieren un tratamiento didáctico mucho más elaborado. Incluso se da la paradoja de que hay educadores que rechazan la concepción positivista del mundo en su manera de entender el tratamiento de los problemas ambientales — se sitúan en la sostenibilidad, en el pensamiento ecológico, en la pedagogía crítica, o en la perspectiva compleja-, pero que son positivistas respecto al modelo de aprendizaje porque adoptan modelos psicoeducativos de corte asociacionista. (García \& Cano, 2006, p.2)

De este modo, el uso de metodologías y estrategias didácticas que componen el modelo pedagógico del docente puede no estar desarrollando en el alumno la suficiente motivación y reflexión para que se empodere de su real condición de agente de cambio ambiental y solo esté transmitiendo conocimientos de la manera tradicional, sin ejercer un conocimiento funcional en donde se revelen las implicaciones de una EA dentro de un marco natural que puede ser transformado con las actuaciones individuales. Entonces, la estructura didáctica que se devela en el aula no tiene la incidencia suficiente para desarrollar en los alumnos pensamientos, actitudes y acciones coherentes. Cuando Escalante (2007) se refiere a este tema es enfático al argumentar que

Hay rutinas y estereotipos bastante alejados de los planteamientos de la teoría de la construcción del conocimiento, que aunque tienen la ventaja de ahorrar tiempo y trabajo, presentan a su vez, una tremenda desventaja: dificultan la reflexión teórica sobre la práctica, y por lo tanto, la mejora de la misma. Así, por ejemplo, existe la creencia que la mera transmisión de información promueve muy diversos aprendizajes, de que hay que tratar de persuadir y convencer a las personas de la bondad de nuestras ideas presentándolas sin más. (Escalante, 2007, p.104)

También existe la creencia de que cualquier modelo vale para cualquier cosa (como si, por ejemplo, comprender el concepto de efecto invernadero fuera lo mismo que aprender la técnica de reciclaje de papel), no se saben en qué momentos y según qué fines es más adecuado basar las estrategias de intervención en modelos asociacionistas o en modelos constructivistas, cuestión que frena el desarrollo de la EA. (Escalante, 2007, p.105).

Algunos afirman que el profesor puede no tener clara la mejor forma de enlazar los objetivos de la educación ambiental y el desarrollo de las actividades propias del área de ciencias naturales y de la institución. escenario que conlleva a realizar muchas labores con poco análisis y reflexión, que por estar relacionadas con el ambiente se suponen que están formando a los alumnos. Convirtiéndose en un dinamismo y activismo de tareas sueltas desde las izadas de bandera, el proyecto de medio ambiente, el PRAE y el trabajo en el aula, pero que no conllevan a una identificación definida del por qué y la convergencia de todas ellas con la evidente consecuencia traumática ya enunciada. La anterior percepción es referenciada por Escalante (2007).

Se realizan un gran número de actividades, pero sin que los participantes tengan claro el sentido de todo aquello, ni controlen el proceso de aprendizaje. [Por lo tanto, es] necesario implicar a los propios ejecutores de la misma [de la educación ambiental], en un proceso de autoconocimiento y autorreflexión, sobre los objetivos y los fines que se persiguen [y] potenciar aún más la realización de trabajos de análisis e investigación sobre sus prácticas [...] potenciar el papel de investigador que pueda desempeñar el educador-monitor encargado de poner en práctica los programas de EA que se diseñan. (Escalante, 2007, p. 105) 
Finalmente, el docente puede no conocer las concepciones de los alumnos, y si las conocen no siempre asume la mejor opción; puede ignorarlas como hace el 99\% de los pedagogos, utilizando los viejos métodos, o puede utilizarlas, permitiendo ante todo su expresión (Giordan \& Souchon, 1997). Como es sabido, el "aprendizaje concreto en muchos casos no se mantiene al cambiar de contexto", cuestión que puede mejorar si se usan estrategias pedagógicas que establezcan relaciones entre las ideas preestablecidas en los alumnos y los conocimientos que la escuela pretende aportar, conduciendo a relaciones entre las ideas elaboradas en ellos, para establecer semejanzas y matizaciones hacia una formulación con un mayor nivel de generalidad (Cano, 2007).

\section{Alumno:}

El alumno como agente principal del proceso educativo es a quien interesa formar para que pueda transformar su realidad y empoderarse de los conocimientos escolares ambientales a través del desarrollo de actitudes, aptitudes y valores que incidan en la preservación del medio ambiente. De hecho el MEN "Ubica al estudiante como centro del proceso educativo con implicaciones no solo pedagógicas sino también culturales, sociales y organizativas, al proporcionarle mecanismos de participación en el propio proceso educativo, en la formulación y adecuación del proyecto educativo institucional y en el gobierno escolar" (MEN, 1994, p.13).

El primer aspecto que se analiza es la importancia de identificar y caracterizar sus necesidades de formación, sus concepciones del mundo y del medio ambiente, sus expectativas de vida, su función como parte de la naturaleza y ciudadano, sus motivaciones, actuaciones y hábitos con el propósito de formular propuestas de formación significativas que logre evidenciar cambios coherentes con la realidad y que se empodere de su entorno para transformarlo. Estas concepciones, según los términos utilizados hoy, tienen una cierta estabilidad y se cree que el aprendizaje de un conocimiento o la adquisición de un procedimiento dependen de ellas. El conocimiento de estas ideas y de estos comportamientos, permite adaptar la enseñanza o al menos proponer una pedagogía mucho más eficaz; es decir, "en cada una de las etapas debe tener en cuenta al estudiante, sus concepciones y su forma de apropiarse del saber." (Giordan \& Souchon, 1997).

Desde estos supuestos, a los niños, niñas y jóvenes parece no interesarles el desarrollo de actitudes y valores pro ambientales relacionados con el agua porque no se identifican como agentes de cambio individuales en problemas ambientales. Esta falta de conciencia de los niños, niñas y jóvenes en la participación en su proceso de formación, y en los cambios que como personas pueden ejercer, hacen que, sin tocar individualidades, se delegue la solución en los otros: el profesor, el padre de familia, las autoridades locales, la parroquia, el Estado; oscureciendo así la posibilidad de hacer cambios que nazcan desde la realidad consciente y personal que puede ser el inicio de grandes cambios. La mirada global que se tiene de esta problemática invade las individualidades y se dejan persuadir de lo innecesario y lo poco que se puede hacer desde lo local o desde lo personal.

Entonces, no existe una formación para la participación ciudadana que considere al individuo "clave en la toma de decisiones públicas en materia de medio ambiente y sostenibilidad". Se olvida que la participación ciudadana permite mejores diagnósticos, un conjunto más rico de posibles soluciones, movilización de recursos, mayor colaboración en la aplicación de las decisiones tomadas, mayor equidad, legitimación y comprensión de decisiones, prevención de conflictos, aprendizaje y mayor integración social (Heras, 2007).

Esta falta de motivación en los alumnos, junto con deficiencias didácticas, puede ser el punto de partida para que estas actividades no permitan desarrollar procesos prácticos y significativos relacionados con la apropiación de conceptos 
ambientales. En contraste, investigaciones han demostrado que "para facilitar la evolución del alumnado entre los niveles de formulación propuestos, es importante trabajar en investigaciones que tengan como base actividades de experiencia directa [...] en situaciones cotidianas y reales", suplementadas con análisis y reflexión de las experiencias empíricas (Cano, 2007).

En primera instancia, la realidad que los alumnos observan en sus hogares y comunidades, y que han sido persistentes a lo largo de toda su vida, están relacionadas con la poca aceptación de proyectos ambientales, el uso inadecuado de recursos naturales, así como el manejo de actividades agropecuarias sin tener en cuenta las consecuencias ambientales. Lo anterior, no permite que aspectos relacionados con la EA sean de importancia para los alumnos pues no se ha evidenciado dentro del cotidiano vivir en sus casas y actividades familiares y mucho menos los beneficios o actitudes de corresponsabilidad.

En segunda instancia, en la escuela se realizan prácticas relacionadas con el medio ambiente pero sin reflexión ni concienciación, tal vez aisladas del contexto, con actividades que no motivan ni inducen actitudes, aptitudes y valores o acciones que generen hábitos y en definitiva no causan mella en su dimensión cognitiva para lograr cambios importantes en sus vidas y mucho menos que empoderen procesos de transformación en sus hogares y/o comunidades. En cuanto al uso del agua se hacen reflexiones en las formaciones generales a manera de regaño por el desperdicio que se observa pero no se han realizado secuencias didácticas dirigidas al reconocimiento de comprensión de conceptos con miras a crear cultura en el uso adecuado del preciado líquido.

Se ha concluido que "el alumnado tiene dificultades para comprender que los asuntos que estamos tratando en clase y la investigación que realizamos son de verdad, no se trata de simulaciones a modo de ejemplo sobre cómo se investiga", imaginarios que se han ido fortaleciendo a través del paso de los años en la escuela, donde no se evidencia la relación de la realidad con lo que pasa en el aula, aspecto ya discutido anteriormente (Cano, 2007).

De esta forma, en el alumno existe una idiosincrasia propia en la que se basan sus pensamientos, supuestos y hábitos y que les hace creer que los cambios individuales no alimentan positivamente las buenas prácticas ambientales de forma global. De este modo, si el alumno no asume con seriedad su responsabilidad social en las cuestiones ambientales difícilmente se lograrán cambios en su actitud y hábitos ecológicos, pues de hecho, se procede de acuerdo a los supuestos que se interiorizan y que definen la actuación humana, el solo discurso no es suficiente aún sustentado con los conocimientos, se requieren otros aspectos de índole reflexivo, crítico y autónomo.

Como se dijo anteriormente, el alumno responde a las condiciones del aula, es por ello, que las características del profesor y su quehacer pedagógico pueden incidir directamente en sus concepciones y si el método y la didáctica no son interesantes para ellos pues se pierden los esfuerzos realizados por la institución o por el mismo profesor en el momento de la apropiación de los contenidos. Tal vez la imposición de tareas académicas de tipo ambiental sean para ellos una carga sin importancia y que cumplen porque es una forma de alcanzar la nota que requieren para pasar el área de ciencias naturales, pero que no generan reflexión suficiente para evidenciar empoderamiento en sus actuaciones.

De esta forma, existen dificultades no solamente conceptuales sino procedimentales, ya que se conciben las tareas escolares y ejercicios que proponen los profesores como formatos estereotipados que persiguen una única respuesta (la correcta) y que no requieren cuestionamiento, así el alumno percibe el trabajo académico como un único camino, con un único resultado y no como la multiplicidad de oportunidades y posibles caminos que pueden generarse cuando se trata de dar respuestas a los interrogantes que se proponen en un contexto real. En otras palabras: 
Los alumnos no superan con facilidad la concepción mecánica y rutinaria de las actividades de clase, de forma que, en un contexto de aprendizaje mucho más abierto, en el que se intenta que investiguen con ayuda de su profesora unos determinados problemas, siguen utilizando las pautas procedimentales aprendidas en el contexto de la enseñanza transmisiva tradicional. Los alumnos interpretan al principio la propuesta de investigación como una tarea escolar más, en la que hay que procurar «adivinar» lo que quiere el profesor para poder «contentarle». (García y Cano, 2006, p. 127)

De otro lado, los alumnos enfrentan dificultades importantes para comprender un determinado proceso y la toma en consideración de variables si el debate solo se sitúa en el plano teórico. Necesita, por tanto, realizar actividades empíricas en las que se manejen las variables propias de que se trata, también se reportan "grandes dificultades para comprender en qué consiste la realización de un plan de trabajo que sirva para comprobar si sus ideas sobre un tema, [...] son más o menos ciertas" (Cano, 2007).

Acompañando a las dificultades conceptuales y procedimentales, la actitudinal también está marcada: la idea de cómo ha de ser un buen escolar como una persona que no interrumpe, no molesta, no pregunta lo primero que se le vienen a la cabeza — pueden reírse de él, reprenderlo- choca con los requerimientos de la tarea investigadora, tarea que necesita de una actitud inquieta, curiosa y creadora, muy alejada del conformismo y la docilidad (Cano, 2007).

Esta situación requiere de la reflexión pedagógica en cuanto que es indispensable reconocer la importancia de los factores relacionados con el alumno; además de los del entorno en la supervivencia del hombre y las otras formas de vida existentes en la Tierra, el grado de culpabilidad humano en el desequilibrio de la naturaleza, así como las formas y prácticas encaminadas al mejoramiento de la situación ambiental. A pesar de que parece un tema global, es evidente que requiere solucionarse en forma regional y local, y sobre todo a nivel individual desde el concepto de corresponsabilidad, porque afecta a todos pero se puede remediar desde la cotidianidad personal y necesita buscar alternativas para crear hábitos, actitudes y valores ambientales en todos los seres humanos como ciudadanos del mundo.

Ahora bien, cuando se reflexiona acerca de las TIC (Tecnologías de la Información y Comunicación) parece ser que los alumnos como nativos digitales tienen acceso a mucha información que por estos medios se maneja, acercando la problemática ambiental a sus vidas de una manera que los motiva. Pero esta información no es suficiente para generar formación ambiental pues no se interioriza, reflexiona o valida en los entornos propios de los niños, niñas y jóvenes, quedando como algo que está pasando en el planeta Tierra pero que como ciudadanos del corriente nada podemos hacer: ¿qué es la Tierra con respecto al universo que existe más allá? En este orden de ideas, las TIC podrían convertirse en herramienta pedagógica que permita establecer algunas didácticas que acerquen los procesos de aprendizaje y enseñanza como alternativa para hacer de los conocimientos una posibilidad de transformación del entorno.

\section{El ámbito de estudio: la educación ambiental}

La EA es considerada en la actualidad como una faceta de la educación formal muy importante y para lograr su comprensión es interesante ahondar en su definición y caracterización. De acuerdo con la UNESCO y el MEN, la educación formal debe cumplir un papel importante en la formación de los niños, niñas y jóvenes colombianos dentro de un panorama de desarrollo humano que permita la preservación del medio ambiente desde una perspectiva sostenible. Es así como dentro de los diversos contextos regionales y locales que atañen a las instituciones educativas, la EA debe cumplir un papel importante para generar en los alumnos actitudes, aptitudes y valores que logren el empoderamiento de los seres humanos en cuanto a una 
relación con el medio ambiente que sea generadora de bienestar social y ambiental.

Como ya se hizo la acotación, la EA en las instituciones educativas de básica primaria y secundaria se ha convertido en una cuestión del área de ciencias naturales que, a través de mecanismos como el currículo, el trabajo en el aula, el PRAE y el proyecto de medio ambiente, debe desarrollar teniendo en cuenta unos lineamientos legales emanados desde la Constitución y el MEN.

La otra apreciación que se ha generado a través de los años, y que tal vez no ha sido objeto de deliberación pedagógica reflexiva, es que la EA se puede delimitar y desarrollar solamente a través de las actividades del proyecto del medio ambiente o del PRAE y los toques esporádicos que se hacen desde algunas asignaturas. Esta situación, unida al activismo relacionado con el medio ambiente a lo largo de los años escolares, ha hecho creer en la supuesta eficiencia de la educación ambiental de las instituciones educativas. De hecho, esta mirada no ha originado mayores inquietudes en la búsqueda de mejores opciones o alternativas didácticas para mejorar la formación ambiental de los alumnos de las instituciones educativas de tipo formal.

Además, la problemática educativa actual no responde a los intereses, necesidades y aspiraciones de tipo medioambiental, productivo y sociocultural, la formación de nuestros niños y jóvenes con patrones de desconocimiento del otro, métodos repetitivos, carencia de afecto, actitudes represivas, coercitivas, agresivas, impositivas, rígidas y normativas, y una pedagogía y un currículo sin sentido, hacen que se forme una muralla entre el maestro y el niño, la escuela y la comunidad, la escuela y la vida (López, 1994).

A pesar de lo anterior, se han reportado investigaciones en donde se evidencian cambios cuando se hacen intervenciones de índole ambiental:

La intervención entre las personas y su entorno puede concretarse entre una triada de transacciones: el nivel del conocimiento y las percepciones, el nivel de las actitudes, creencias y valores y el nivel de los comportamientos, es en todos estos niveles en los que pretende incidir nuestro trabajo [educación ambiental] y esta investigación parece demostrar que la educación ambiental es una herramienta eficaz para trabajar simultáneamente en estos aspectos y que se logran cambios significativos en todos ellos, tanto en los niños como en los adultos. (Navarro, 2007, p.46)

En todo caso, es necesario mejorar la situación ambiental actual teniendo en cuenta que el hombre, como la única forma de vida pensante, tiene la responsabilidad moral de hacerlo y se ha demostrado a través de los años, que sus actos parecen carecer de una falta de proyección en esta área del conocimiento, lo cual se evidencia en la disminución de la calidad de vida humana y degradación de las demás formas de vida que existen, de tal forma que no ha sido posible mantener un equilibrio entre la naturaleza, sus procesos naturales y las actividades humanas. En consecuencia, el ciclo es vicioso y los cambios negativos que se han evidenciado en el medio ambiente están repercutiendo directamente en la salud humana, en sus actividades y su idiosincrasia, haciendo urgente la reflexión y el empoderamiento para lograr cambios amigables con el entorno y con este en la calidad de vida humana.

\section{Las políticas y normatividad}

La Constitución 1991 acogen disposiciones internacionales como la obligatoriedad del Estado y de las personas de proteger las riquezas culturales y naturales de la Nación; y declara los derechos colectivos y del ambiente en donde estipula el derecho de las personas a gozar de un ambiente sano, la participación de la comunidad en las decisiones que puedan afectarlo, el deber del Estado de proteger la diversidad e integridad del ambiente, conservar las áreas de especial importancia ecológica y fomentar la educación para el logro de estos fines. Cuando habla específicamente de la 
educación, enuncia la formación del colombiano para el mejoramiento cultural, científico, tecnológico y para la protección del ambiente siendo la EA una de las estrategias para reducir las tendencias actuales de destrucción y el desarrollo de una nueva concepción de la relación social-naturaleza. (MEN, 1994, p. 101).

Es así como la política pública colombiana, desde el marco de disposiciones normativas pretende mejorar procesos de EA, como el "objetivo fundamental de cerrar las brechas de inequidad recogido en el Plan Nacional de Desarrollo 20102014 Camino a la Prosperidad propende hacia el mejoramiento de la calidad de la educación y la formación para el ejercicio de la ciudadanía constituyen la herramienta fundamental para formar a las ciudadanas y ciudadanos en la capacidad de contribuir a los procesos de desarrollo cultural, económico, político y social y en la sostenibilidad ambiental; en el ejercicio de una ciudadanía activa, reflexiva, crítica y participativa, que conviva pacíficamente y en unidad, como parte de una nación próspera, democrática e incluyente. (MEN, 2013, p. 5)

\section{Por su parte, el MEN de Colombia}

Plantea que la educación ambiental debe ser considerada como un proceso que permita al individuo comprender las relaciones de interdependencia con su entorno, a partir del conocimiento reflexivo y crítico de su realidad biofísica, social, política, económica y cultural para que, a partir de la apropiación de la realidad concreta, se puedan generar en él y en su comunidad actitudes de valoración y respeto por el ambiente. (RED, 2000, p. 85)

Teniendo en cuenta a lo anterior, Colombia ha establecido normatividad en cuanto a EA, desde que en 1994 se expidió el Código Nacional de los Recursos Naturales y Renovables y de Protección del Medio Ambiente, que se reglamentó mediante el Decreto 1337 de 1978, en donde refería la EA con perspectiva conservacionista y su implementación a través de cursos de ecología, de preservación ambiental y de recursos naturales, impulsando solamente jornadas ecológicas en los planteles educativos (RED, 2000, p. 92).

Posteriormente, el MEN desarrolló una propuesta de EA, iniciando con una exploración para identificar los obstáculos, una etapa de profundización para la formulación de unos lineamientos de política que involucrara a los diferentes actores con énfasis en el sector escolar, y finalmente una etapa de proyección con la pretensión de realizar la difusión de los Lineamientos de Política de Educación Ambiental (RED, 2000, p. 97). En este marco, la intención del MEN, a través del Programa de Educación Ambiental, es la de propiciar espacios de reflexión y de intercambio para las conceptualizaciones, metodologías y estrategias, propiciar espacios para la teoría pero también para la praxis, para el seguimiento pero también para la evaluación formativa. Es así como en la actualidad existen algunas estrategias que hacen parte de las políticas educativas nacionales: coordinación intersectorial e interinstitucional, inclusión de la dimensión ambiental en la educación formal (PRAES — Proyectos Ambientales Escolares-), no formal (PROCEDAS - Proyectos Ciudadanos de Educación Ambiental-) e informal, participación ciudadana, investigación, formación de educadores ambientales, diseño, implementación, apoyo y promoción de planes y acciones de comunicación y divulgación, promoción y fortalecimiento del Servicio Militar Ambiental, promoción de la etnoeducación en la Educación Ambiental e impulso a proyectos ambientales con perspectiva de género (RED, 2000, p. 108).

Como resultado de este programa, el MEN en su documento de lineamientos para la política nacional ambiental:

Plantea como base para una Educación Ambiental, [que] el medio ambiente ha de ser visto como un sistema dinámico definido por las interacciones físicas, biológicas, sociales y culturales percibidas 
o no por los seres humanos y demás seres vivos y los elementos del medio en el cual se desenvuelven, bien sean de carácter natural o transformados o creados por el hombre (Torres y Cotes 1998). (RED, 2000, p. 77)

A pesar de lo anterior, no se ha evidenciado una EA en las instituciones educativas que dirijan sus actividades hacia la formación de ciudadanos y ciudadanas que con sus actitudes, aptitudes y valores ecológicos logren una relación equilibrada y sana con el ambiente en el cual viven. Aunque es importante recalcar que desde la normatividad colombiana existen unos pilares fuertes y firmes a partir de los cuales se pueden recrear posibilidades y estrategias para lograr los objetivos de formación en educación ambiental tan anhelados.

\section{Referencias bibliográficas}

Álvarez, D. (2012). Diseño de material didáctico para la enseñanza de las ciencias naturales. Aplicado a la conservación del agua en zona de influencia de la I. E. R. El Tambo. (Antioquia, Colombia). Trabajo de investigación como requisito parcial para optar al título de Magister en la enseñanza de las ciencias exactas y naturales. Universidad Nacional de Colombia, Medellín.

Antoraz, M. y Martinez, F. (2007). El agua y la educación medioambiental hacia una nueva cultura del agua en la escuela. Ponencia en Congreso Agua y Educación Ambiental: nuevas propuestas para la educación.

Astolfi, J. (2001). Conceptos clave en la didáctica de las disciplinas. Referencias, definiciones y bibliografías de didáctica de las ciencias. SeviIla, España: Diada Editora S.L.

Badia, R. (2010). El modelo sistémico complejo como herramienta de análisis de un material didáctico en el marco de una investigación-acción: el caso del Camp D’aprenentatge de les valls D aneu. En: Doctorado Interuniversitario en Educación Ambiental. Investigar para avanzar en educación ambiental (pp. 32-43). Barcelona: Organismo Autónomo Parques Nacionales.

Barrero, D. (2012). Valle de Laciana: diagnóstico de la situación ambiental, propuesta de acciones educativo-ambientales para una comarca en reconversión. En: Doctorado Interuniversitario en Educación Ambiental. Nuevas Investigaciones Iberoamericanas en educación ambiental (pp. 147-165). Barcelona: Organismo Autónomo Parques Nacionales.

Barros, J. (2012). La cooperación internacional en educación ambiental: un análisis de la experiencia de la comunidad de los países de lengua portuguesa (CPLP) en la organización de la conferencia internacional infantojuvenil (CONFINT). En: Doctorado Interuniversitario en Educación Ambiental. Nuevas Investigaciones Iberoamericanas en educación ambiental (pp. 25-30). Barcelona: Organismo Autónomo Parques Nacionales.

Bozic, M. (2010). Comunicación de sostenibilidad a través del arte. Estudios de caso de Dah Teatro y Hundertwasser. En: Doctorado Interuniversitario en Educación Ambiental. Investigar para avanzar en educación ambiental (pp. 242-254). Barcelona: Organismo Autónomo Parques Nacionales.

Cardona, M. (2002). Introducción a los métodos de investigación en educación. Universidad de Alicante. Madrid: Editorial EOS.

Carr, W. y Kemmis, S. (s.f.). Investigación - acción. Notas tomadas de "Action reserch as a form of staff development in higher Education". David Kember y Lyn Gow. Higher Education 23, 297 310, 1992. Traducido por Pedro D. Lafourcade.

Carrasco, M. T: (1994). Dimensión ambiental y proceso educativo: una propuestas y un propósito ambientales. En: Ministerio de Educación Nacional. (1994). Memorias Serie documentos especiales MEN. Seminario Internacional La dimensión ambiental y la escuela (pp. 97-119). Bogotá: Papelgrafico. 
Comité Técnico Interinstitucional de educación ambiental del Valle de Cauca. (2008). Lineamientos generales de política de educación ambiental para el Valle del Cauca. Santiago de Cali. Recuperado de www.valledelcauca.gov. co/educacion/descargar.php?id=4979. $\otimes$

Constitución Política de Colombia de 1991. Recuperado de http://www.alcaldiabogota.gov.co/ sisjur/normas/Norma1.jsp?i=4125.

Chamorro, P. (2007). La formación de naturalistas como aporte a la conservación de la biodiversidad. Estudio de caso de la escuela argentina de naturalistas. Ibrahim, A. et. al. Tendencias de la Investigación en Educación Ambiental al desarrollo socioeducativo y comunitario (pp. 134-163). Organismo Autónomo Parques Nacionales, Ministerio del Medio Ambiente.

Declaración de la Conferencia Intergubernamental de Tbilisi sobre educación ambiental. (1977) (Tbilisi, Georgia, 14-26 de octubre de 1977). Recuperado de http://platea.pntic.mec.es/ jpascual/eama/tbilisi.html.

Decreto 1743 de 1994. Recuperado de http:// www.minambiente.gov.co/documentos/ dec 1743 030894.pdf 25 de marzo 2014

Decreto 1860 de agosto 3 de 1994. Recuperado de http://www.mineducacion.gov.co/1621/articles-86240 archivo pdf.pdf.

Declaración de Rio de Janeiro. Recuperado de http://siga.jalisco.gob.mx/assets/documentos/ TratadosInt/DeclaraRio 92.htm.

Doctorado Interuniversitario de Educación Ambiental. (2010). Investigar para avanzar en Educación Ambiental. Naturaleza y Parques nacionales. Serie educación ambiental. Barcelona: Editorial Organismo Autónomo Parques Nacionales. Ministerio de Medio Ambiente y Medio Rural y Marino.

Elliot, J. (2000). La investigación-acción en Educación. Cuarta edición. Ediciones Morata. Recuperado de http://plataforma.upnmazatlan. com/sites/default/files/180/ELLIOT\%20John. pdf.
EOT Municipal Choachi Cundinamarca. Recuperado de http://www. choachi-cundinamarca.gov.co/apc-aa-files/30363665623037386465386665383333/ EOT CHOACHI.pdf.

Equipo de trabajo de la experiencia. (1994). Propuesta pedagógica para el desarrollo local ambiental, experiencia del municipio de Planeta Rica. En: Ministerio de Educación Nacional. (1994). Memorias Serie documentos especiales MEN. Seminario Internacional La dimensión ambiental y la escuela (pp. 205- 210). Bogotá, Colombia: Papelgrafico.

Escalante, A. (2007). Hacia una caracterización de modelos de actuación de monitores: dos estudios de caso desde la perspectiva constructivista. En: Ibrahim, A. et. al. Tendencias de la Investigación en Educación Ambiental al desarrollo socioeducativo y comunitario (pp. 102-114). Organismo Autónomo Parques Nacionales, Ministerio del Medio Ambiente.

España, C. (2008). El agua viajera. En: Estándares en el Aula. Relatos docentes. Ministerio de Educación Nacional de Colombia, Bogotá. Recuperado de hhttp://www.colombiaaprende.edu. co/html/micrositios/1752/articles-327868 lecturas 4.pdf.

Fernández, A. (2012). Análisis de la dimensión ambiental en la educación para el desarrollo de la cooperación internacional. En: Doctorado Interuniversitario de Educación Ambiental. Nuevas Investigaciones Iberoamericanas en educación ambiental (pp. 229-248). Barcelona: Organismo Autónomo Parques Nacionales. Fernández, D. (2003, noviembre). De la teoría a la práctica en educación ambiental: el caso del agua. Ponencia marco Ponencias y comunicaciones presentadas en el Congreso Agua y Educación Ambiental: Nuevas Propuestas para la Acción. Alicanate.

Fernández, J. (2012). La construcción del conocimiento sobre la gestión y la contaminación del agua. Concepciones del alumnado de primero de bachillerato. Tesis doctoral. Facultad 
ciencias de la educación. Universidad de Sevilla, España.

Fernández, R. (2010). El astillero y la educación ambiental: un proyecto para fomentar la motivación sobre su entorno natural de los niños(as) y jóvenes de Melchor Ocampo, Veracruz (México). En: Doctorado Interuniversitario en Educación Ambiental. Investigar para avanzar en educación ambiental (pp. 85-101). Barcelona: Organismo Autónomo Parques Nacionales.

Fonseca, L. (2007). Educación ambiental a la luz de Paulo Freire: un estudio con los profesores de la red municipal de enseñanza de diferentes ecosistemas del estado de Ceará. En: Ibrahim, A. et. al. Tendencias de la Investigación en Educación Ambiental al desarrollo socioeducativo y comunitario (pp. 85-101). Organismo Autónomo Parques Nacionales, Ministerio del Medio Ambiente.

Fontes da Costa, S. (2012). La integración de las dimensiones cultural y biofísica de las zonas costeras en los equipamientos de educación ambiental: un estudio exploratorio sobre el potencial y la práctica en la región del Eixo Atlántico. En: Doctorado Interuniversitario de Educación Ambiental. Nuevas Investigaciones Iberoamericanas en educación ambiental (pp. 49-66). Barcelona: Organismo Autónomo Parques Nacionales.

García, J. (1998). Hacia una teoría alternativa sobre los contenidos escolares. Sevilla: Diada Editora S. L.

García, J. y Cano, M. (2006). ¿Cómo nos puede ayudar la perspectiva constructivista a construir conocimiento en Educación Ambiental? Revista Iberoamericana de Educación (41), 117-131. Recuperado de: http://www.rieoei. org/rie41a05.pdf.

García, M. (2010). El modelo sistémico complejo como instrumento de análisis del grado de complejidad de las actividades del campo de aprendizaje de los monasterios de Cister. En: Doctorado Interuniversitario de Educación Ambiental. Investigar para avanzar en educación ambiental (pp. 102-120). Barcelona: Organismo Autónomo Parques Nacionales.

Giordan, A y Souchon, C. (1997). La educación ambiental: guía práctica. (Segunda edición). Sevilla: Diada Editora S. L.

Gómez, M. (2012). Evaluación del programa escuelas verdes en Guanajuato. Experiencias en Educación Ambiental en los Centros Educativos Participantes en 2001 y 2001 (1 generación). En: Doctorado Interuniversitario de Educación Ambiental. Nuevas Investigaciones Iberoamericanas en educación ambiental (pp. 317-337). Barcelona: Organismo Autónomo Parques Nacionales.

González, C. (2012). Aplicación del constructivismo social en el aula. Instituto para el desarrollo y la innovación educativa en educación bilingüe y multicultural —IDIE_- Organización de estados iberoamericanos para la educación la ciencia y la cultura - OIE — oficina de Guatemala. Recuperado de: http://www.oei. es/formaciondocente/materiales/OEI/2012_ GONZALEZ_ALVAREZ.pdf.

González, F. (2011). El medio urbano como ámbito de conocimiento escolar. Análisis y propuestas a partir de un estudio de concepciones de alumnado de Bogotá. Tesis doctoral. Facultad de Ciencias de la Educación. Departamento de Didáctica de las Ciencias Experimentales y Sociales. Universidad de Sevilla. Sevilla, España.

González, F. (2014). Comunicación personal.

Guzman, R. et. al. (2008). Análisis de la investigación cualitativa en educación desarrollada en el estado español. Revista Qurriculum (21), 157-184. Recuperado de http://publica.webs. ull.es/upload/REV\%20QURRICULUM/21\%20 -\%202008/07\%20Guzm\%C3\%A1n\%20 y\%20otros.pdf.

Gutiérrez, J. (1995). La educación ambiental. Fundamentos teóricos, propuestas de transversalidad y orientaciones extracurriculares. Madrid: Editorial La Muralla S.A.

Heras, F. (2007). La participación como proceso de aprendizaje y conocimiento social: la 
participación pública en la evaluación de Impacto ambiental del embalse de Bernardos (Segovia). En: Ibrahim, A. et. al. Tendencias de la Investigación en Educación Ambiental al desarrollo socioeducativo y comunitario. (pp. 269-287). Organismo Autónomo Parques Nacionales, Ministerio del Medio Ambiente.

Heredia, M. A. (2010). El rio Guadalete como recurso educativo en el aula. Análisis de las dificultades de aprendizaje detectadas mediante un cuestionario de detección de ideas basado en imágenes. En: Doctorado Interuniversitario de Educación Ambiental. Investigar para avanzar en educación ambiental (pp. 121-140). Barcelona: Organismo Autónomo Parques Nacionales.

Hernández, S. (2008). El modelo constructivista con las nuevas tecnologías: aplicado en el proceso de aprendizaje. Revista de Universidad y Sociedad del Conocimiento 5 (2), 26-36.

Horita, L. (2012). Estudio de las representaciones sociales de la educación ambiental en estudiantes de la universidad intercultural de Chiapas. En: Doctorado Interuniversitario de Educación Ambiental. Nuevas Investigaciones Iberoamericanas en educación ambiental (pp. 339-357). Barcelona: Organismo Autónomo Parques Nacionales.

Ibarra, J. (2007). Nuevos contenidos educativos sobre el agua y los ríos desde una perspectiva CTS. Revista electrónica de enseñanza de las ciencias (6), 714-728. Recuperado de http://reec.uvigo.es/volumenes/volumen6/ ART13 Vol6 N3.pdf

Ibrahim, A. (2007). Competencias y recursos de comunicación social para un desarrollo local sostenible. Diagnóstico y perspectivas, el caso de Marruecos. En: Ibrahim, A. et. al. Tendencias de la Investigación en Educación Ambiental al desarrollo socioeducativo y comunitario. (pp. 249-268). Organismo Autónomo Parques Nacionales, Ministerio del Medio Ambiente.

Jiménez, A. (2010). Diagnóstico de la calidad de los equipamientos urbanos en educación ambiental. Estudio de casos dela comunidad de Madrid. En: Doctorado Interuniversitario en Educación Ambiental. Investigar para avanzar en educación ambiental (pp. 255-287). Barcelona: Organismo Autónomo Parques Nacionales.

Kember, D. y Gow, L. (1992). Investigación acción. Notas tomadas de "Action reserch as a form of staff development in Higher Education". Higher Education (23), 287-310. Kluwer Academic Press Publisher. Netherlands.

Kong, F. (2010). La visión de futuro de niños y niñas de 6 de primaria: un caso de estudio en una escuela de Barcelona. En: Doctorado Interuniversitario en Educación Ambiental. Investigar para avanzar en educación ambiental (pp. 141-304). Barcelona: Organismo Autónomo Parques Nacionales.

Ley General Ambiental de Colombia. Ley 99 de 1993. Diario Oficial No. 41.146, de 22 de diciembre de 1993. Recuperado de http://www. minambiente.gov.co/documentos/normativa/ ley/ley 0099 221293.pdf.

Ley 115 de febrero 8 de 1994. Recuperado de http://www.mineducacion.gov.co/1621/articles-85906 archivo pdf.pdf.

Llerena, G. (2010). Estudio de caso para la evaluación de una agenda 21 escolar municipal: el caso de Sant Cugat del Vallès. En: Doctorado Interuniversitario en Educación Ambiental. Investigar para avanzar en educación ambiental (pp. 160-179). Barcelona: Organismo Autónomo Parques Nacionales.

López, A. (1994). La educación ambiental, un modo de vida, experiencia de la corporación maestra vida en el Tambo, Cauca. En: Ministerio de Educación Nacional. (1994). Memorias Serie documentos especiales MEN. Seminario Internacional La dimensión ambiental y la escuela (pp. 187-195). Bogotá: Papelgráfico.

Magin, C. (2012). La educación para la sostenibilidad dentro de la educación en el tiempo libre. En: Doctorado Interuniversitario de Educación Ambiental. Nuevas Investigaciones 
Iberoamericanas en educación ambiental (pp. 86-106). Barcelona: Organismo Autónomo Parques Nacionales.

Marcen, C. (2003). Aportaciones desde la escuela a la nueva cultura del agua. Ponencia en el Congreso Agua y Educación Ambiental: nuevas propuestas para la acción. Recuperado de http://www.ciceana.org.mx/recursos/tribunatura/pdf/Agua y educacion ambiental. pdf.

Márquez, D. (2003). De la teoría a la práctica en educación ambiental: el caso del agua. Ponencia marco Congreso Agua y Educación Ambiental: nuevas propuestas para la acción. Pp. 13-21 Recuperado de http://www.ciceana. org.mx/recursos/tribunatura/pdf/Agua y educacion ambiental.pdf.

Martínez, A. (2007). La educación ambiental y la formación profesional ocupacional: evaluación del desarrollo y resultados de la integración y enseñanza del módulo de sensibilización ambiental. Estudio de un caso. En: Ibrahim, A. et. al. Tendencias de la Investigación en Educación Ambiental al desarrollo socioeducativo y comunitario (pp. 115-133). Organismo Autónomo Parques Nacionales, Ministerio del Medio Ambiente

Ministerio de Educación Nacional. Serie lineamientos curriculares. Recuperado de http:// www.mineducacion.gov.co/cvn/1665/articles-89869 archivo pdf5.pdf.

Ministerio de Educación Nacional. (1994). Memorias Serie documentos especiales MEN. Seminario Internacional La dimensión ambiental y la escuela. Bogotá: Papelgráfico.

Ministerio de Educación Nacional. (2013), Guía 49. Guías pedagógicas para la convivencia escolar. Ley 1620 de 2013 - Decreto 1975 de 2013. Bogotá: Amado Impresores S.A.S.

Navarro, L. (2007). Educación ambiental y conservación de murciélagos en Hidalgo, México. En: Ibrahim, A. et. al. Tendencias de la Investigación en Educación Ambiental al desarroIlo socioeducativo y comunitario (pp. 35-51).
Organismo Autónomo Parques Nacionales, Ministerio del Medio Ambiente.

Noguera, E. (2012). Sostenibilidad y educación infantil: un estudio de caso para promover la sensibilización socio-ambiental en la primera etapa de la escolarización. En: Doctorado Interuniversitario de Educación Ambiental. Nuevas Investigaciones Iberoamericanas en educación ambiental (pp. 107-127). Barcelona: Organismo Autónomo Parques Nacionales.

Oliveira, M. (2012). Educación para la sustentabilidad en la enseñanza superior: concepciones de los profesores del curso de pedagogía de una facultad de Vitoria-Espirito Santo-Brasil. En: Doctorado Interuniversitario de Educación Ambiental. Nuevas Investigaciones Iberoamericanas en educación ambiental (pp. 398-417). Barcelona: Organismo Autónomo Parques Nacionales.

Organización de las Naciones Unidas (Junio, 1972). Declaración de Estocolmo sobre el medio ambiente humano. Adopción: Conferencia de las Naciones Unidas sobre el Medio Ambiente Humano. Recuperado de http://www. ordenjuridico.gob.mx/TratInt/Derechos $\% 20$ Humanos/INST\%2005.pdf.

Organización de las Naciones Unidas para la Educación, la Ciencia y la Cultura UNESCO (1977). Informe final Conferencia Intergubernamental de Educación Ambiental, en Tbilisi, Georgia, para octubre de 1977. Recuperado de http://unesdoc.unesco.org/images/0003/000327/032763sb.pdf.

Pardo, F. (1996). Quinientos años de historia chiguana. Litoformas Modelo: Santa Fé de Bogotá, Colombia.

Programa de las Naciones Unidas para el Desarrollo. (2011). Colombia rural. Razones para la esperanza. Informe Nacional de DesarroIlo Humano. Recuperado de http://planipolis. iiep.unesco.org/upload/Colombia/Colombia NHDR 2011.pdf.

Programa de Fortalecimiento de la Capacidad Científica en la Educación Básica y Media 
RED. Universidad Nacional de Colombia. (Junio, 2000). Educación Ambiental: Un estado del arte. En: Interdisciplinariedad y currículo: construcción de proyectos escuela-universidad. Memorias del V seminario internacional Bogotá. Recuperado de http://www.bdigital. unal.edu.co/1411/2/01PREL01.pdf.

Quintero, J., Munévar, R. y Yepes, J. (2007). Investigación-acción y currículo: un recorrido por el mundo. latinoam.estud.educ. 3 (1),123- 142.

Ramírez, N. (2012). Análisis del discurso ecológico del espacio europeo de educación superior. En: Doctorado Interuniversitario de Educación Ambiental. Nuevas Investigaciones Iberoamericanas en educación ambiental. (pp. 130-146. Barcelona, España: Organismo Autónomo Parques Nacionales.

Santiváñez, V. (s.f.). La didáctica, el constructivismo y su aplicación en el aula. Pp. 137-148. Recuperado de: http://www.fcctp.usmp.edu. pe/cultura/imagenes/pdf/18_07.pdf.

Solís, M. (2007). Estudio de viabilidad para desarrollar una red de educación ambiental en el Parque Natural Sierra Norte de Sevilla. En: Ibrahim, A. et. al. Tendencias de la Investigación en Educación Ambiental al desarrollo socioeducativo y comunitario (pp. 164-181). Organismo Autónomo Parques Nacionales, Ministerio del Medio Ambiente.

Suarez, A. (2012). Concepciones de los profesores sobre la dimensión ambiental en los estudios superiores: el caso de la titulación de trabajo social en la Universidad del Quindío Colombia. En: Doctorado Interuniversitario en Educación Ambiental. Nuevas Investigaciones Iberoamericanas en educación ambiental (pp. 530-556). Barcelona: Organismo Autónomo Parques Nacionales.

Toledano, M. (2012). Educación ambiental y decrecimiento. Análisis de las prácticas de un colectivo (el enjambre sin reina). En: Doctorado
Interuniversitario en Educación Ambiental. Nuevas Investigaciones Iberoamericanas en educación ambiental (pp. 557-575). Barcelona: Organismo Autónomo Parques Nacionales.

Toledano. M. A. (2012). Ecología a escala escolar: Una propuesta didáctica de educación acción con la cual incorporar empíricamente diversos aspectos teóricos sugeridos para hacer educación ambiental. En: Doctorado Interuniversitario en Educación Ambiental. Nuevas Investigaciones Iberoamericanas en educación ambiental (pp. 577-594). Barcelona: Organismo Autónomo Parques Nacionales.

Torres, M. (1992). La Educación Ambiental en Colombia: Un proceso construido a la luz de una mirada investigativa. Programa de Educación Ambiental, Ministerio de Educación Nacional_Universidad Distrital, Bogotá (Colombia).

Tristán, M. (2010). Las concepciones en educación ambiental de los docentes de educación primaria obligatoria en el distrito de Sonà, Panamá. En: Doctorado Interuniversitario en Educación Ambiental. Investigar para avanzar en educación ambiental (pp. 201-220). Barcelona: Organismo Autónomo Parques Nacionales.

Vitorassi, S. (2012). Construcción participativa de indicadores del programa de educación ambiental de Itaipu binacional. En: Doctorado Interuniversitario en Educación Ambiental. Nuevas Investigaciones Iberoamericanas en educación ambiental (pp. 5945-612). Barcelona: Organismo Autónomo Parques Nacionales. Zegarra, A. (2007). Preconcepciones y valoración de los especialistas y docentes de educación de los diferentes niveles de formación básica de la región Puno - Perú, sobre la educación ambiental y la Puna. En: Ibrahim, A. et. al. Tendencias de la Investigación en Educación Ambiental al desarrollo socioeducativo y comunitario (pp. 52-70). Organismo Autónomo Parques Nacionales, Ministerio del Medio Ambiente. 